\title{
AdS/CFT Duality and the Emergence of Spacetime
}

\author{
Dean Rickles
}

Unit for HPS, University of Sydney

\begin{abstract}
The AdS/CFT duality has been a source of several strong conceptual claims in the physics literature. In this paper I focus on one of these: the extent to which spacetime geometry and locality can be said to emerge from this duality, so that neither is fundamental. I argue: (1) that the kind of emergence in question is relatively weak, involving one can of spacetime emerging from another kind of spacetime; (2) inasmuch as there is something conceptually interesting to say about the emergence of spacetime and locality (vis-à-vis spacetime ontology), it is no different from that already well known to those within canonical quantum gravity; (3) that at the core of AdS/CFT is an issue of representation and redundancy in representation.
\end{abstract}

Key words: AdS/CFT Correspondence, Emergence, String theory, Duality, Representation.

PACS: 04.50.-h, 11.25.Mj, 11.25.-w, 01.70.+w, 02.40.-k, 11.30.Ly, 11.25.Hf, 11.15.Me

The richness of "emergent" phenomena that are hiding behind equations as simple as the gauge theory Lagrangian - especially in the "modern" regime when the number of colors and/or the coupling constant is large -is one of the most important conceptual insights of the last decade in theoretical physics.

Luboš Motl

Email address: dean.rickles@sydney.edu.au (Dean Rickles).

1 I gratefully acknowledge the Australian Research Council for the award of an Australian Research Fellowship which enabled me to complete this work. 


\section{An Emerging Theme in the Philosophy of Spacetime Physics}

Just when philosophers were getting to grips with the implications of general relativity, they must now face the question of whether it is even a valid description at microscopic distances or simply an effective description that applies at (relatively) low energies. If it does indeed fail to hold at microscopic distances, then what is it replaced by? The matter is a particularly pressing one for philosophers of spacetime physics since we are now used to thinking of spacetime geometry and gravitation as inseparable. Does the failure of general relativity, then, also imply the breakdown of spacetime geometry itself at microscopic distances? Again, if so, what description is it replaced by? Further, how do we achieve the apparently smooth, macroscopic picture of spacetime of general relativity from whatever operates at the microscopic level? Also, without a fundamental spacetime, how do we recover the apparently localized observables that we find in low energy situations?

One answer is provided by the AdS/CFT correspondence (or, more accurately, conjecture), discovered within the general framework of string theory. In a nutshell, this involves a duality (that is, an exact physical equivalence) between a quantum mechanical theory without gravity (a Yang-Mills theory) and a quantum mechanical theory with gravity (a closed string theory). This equivalence is achieved by defining the theories within spacetimes of different dimensionality. There seems to be a general belief amongst physicists working in this area that, given the deep relationship between gravity and geometry, the correspondence implies emergent spacetime and locality. More specifically: a very particular kind of spacetime (one that provides a background for a very particular kind of string theory) is said to emerge from a very particular kind of (dual) gauge theory living on a lower dimensional space that forms the boundary lining the spacetime of the string theory. One can study the (quantum gravitational) physics of the interior of this lining by doing (non-gravitational) physics on the lining alone, using a quantum gauge field theory. The curved spacetime of general relativity is, then, viewed as a kind of holographic construct from a lower dimensional physics (without curved spacetime) rather than a fundamental feature of reality. ${ }^{2}$ It is the purpose of this note to probe this confusing and ontologically weighty implication.

We begin in the next section with a highly condensed presentation of some central facts of superstring theory that will be needed to understand the rest of the paper. In section 3 we introduce the more general principle of holography and the related gauge/string duality. Then in section 4 we get to grips with the specialised case of AdS/CFT duality. Section 5 will focus on the precise sense

$\overline{2}$ Polyakov (1999) likens this situation to the inhabitants of Plato's cave assuming that the shadows are real, rather than projections from a more fundamental reality. 
in which spacetime and locality are thought to emerge within AdS/CFT and will aim to show that, inasmuch as there is emergence at all, it is on all fours with that found in, e.g., loop quantum gravity and other gravitational theories with a vanishing Hamiltonian, including Feynman quantization. To conclude, in section 6, I turn briefly to issues of representation and redundancy.

\section{Superstring Theory}

The standard capsule definitions of superstring theory note how point-particles are transformed into excitations of one-dimensional (open or closed) strings propagating in a ten-dimensional Minkowski spacetime. Many of the most important features of string theory are consequences of the fact that the trajectory of such one-dimensional objects through spacetime generates a twodimensional Riemann surface (the worldsheet or worldtube). ${ }^{3}$ The dynamics is given by an action that demands that the worldsheet surface area be kept to a minimum. The splitting and joining of these strings, determined by the string coupling constant, is able to reproduce the predictions of general relativity at low energies (i.e. the perturbative expansion of general relativity) but gives new physics at higher energies. One can develop a quantum string theory by applying a version of Feynman quantization to this classical system so that one sums over worldsheets. ${ }^{4}$

This much provides the perturbative definition of string theory, for which the string coupling constant $g_{s}$ is small. The non-perturbative story remains vague. However, the AdS/CFT duality is most likely a crucial part of the story and offers otherwise missing insights into (non-perturbative aspects of) string theoretic quantum gravity. Also central to the non-perturbative story is the distinction between closed and open strings: the former involve gravity, the latter do not (though they do involve gauge fields). When open strings are present, boundaries need to be present for them to end upon. These boundaries, known as $\mathrm{D} p$-branes (where the ' $\mathrm{D}$ ' stands for the Dirichlet boundary conditions at the end points of strings and the $p$ provides the dimensionality of the brane), have a dynamics of their own and are closely connected to, what are in string theory, hard to model phenomena involving strong gravitational fields, such as black holes - they lie at the heart of the duality between gravity

$\overline{3}$ The string history is given by a map $X^{\mu}(\sigma, \tau)$ from this worldsheet (with coordinates $(\sigma, \tau)$ into spacetime $X^{\mu}$.

4 In fact, the path integral (known as the Polyakov integral) ranges over the moduli spaces of the Riemann surface representing the worldsheet. The purely topological nature of the expansion means that there is only one diagram at each order, with any diagrams with the same number of handles being homeomorphic to one another. 
and gauge theory. ${ }^{5}$

D-branes have a tension that is inversely proportional to the string coupling constant describing the strength of interactions; therefore, at weak coupling (i.e. in the perturbative expansion for which $g_{s} \ll 1$ ) they will be unobservable. ${ }^{6}$ (It is crucial to note that this tension contributes to the stress-energy tensor and therefore can warp spacetime geometry in the vicinity of the Dbrane.) Strings can also be bounded by pairs of D-branes (see fig.1) and when this happens the strings become massless (and are able to mimic gluons) - the open string excitation spectrum contains a massless spin-1 particle, so that individual $\mathrm{D} p$-branes with open strings attached will support a $U(1)$ gauge field on its $(p+1)$-dimensional world-volume (the open strings, in 1st excitation, will be described by a $(p+1) \mathrm{D} U(1)$ gauge theory). When there are $N$ parallel D-branes (with strings held between any pair of them) the gauge group is amplified up to $U(N)$ (so that the open strings will be described by a $U(N)$ gauge theory). The gauge theoretic aspects arise from the fact that the degrees of freedom on the brane are matrix valued, where the indices of the matrix $M_{i j}$ refer to the endpoints of the open string (if the endpoints lie on different branes then $i \neq j$ ).

For large $N$ the D-brane stack becomes heavy enough to form an event horizon, and is known as a black brane and will be part of a theory of closed strings (i.e. with gravity). This multiplicity in languages to describe the physical situation of $\mathrm{D} p$-branes (gauge fields on worldvolumes versus objects embedded in string theory backgrounds) forms the core of the gauge-string dualities and is utilized in the AdS/CFT correspondence.

As is now well known, there are five consistent (Lorentz invariant) superstring theories (propagating in 10 dimensional spacetime). These theories differ with respect to their chirality, gauge groups, supersymmetry generators, heterotic type (whether or not they contain bosonic and superstrings), and whether or

$\overline{5}$ Just as strings trace out worldsheets, Dp-branes trace out $p+1$-dimensional worldvolumes. In fact, a string can be viewed as a kind of low-dimensional brane, a 1-brane (or D1-brane). Likewise, a particle or point-like black hole can be understood as a D0-brane. We will be interested in D3-branes and their four-dimensional worldvolumes. There is a model (the Randall-Sundrum model: see Randall and Sundrum (2011)) according to which our universe is represented by the worldvolume of a D3brane embedded in $A d S_{5}$ (to be discussed below)), with the strong and electroweak forces localized on the brane, and gravity able to propagate off the brane (in the so-called "bulk space").

6 Though they will still have the description as a surface (in the full 10 dimensional spacetime) on which open strings are confined. Closed strings are free to move away from this surface (in the bulk). If open strings join to form a closed string then they too can move off the D-brane, and this corresponds to gravitational radiation being emitted from a photon. 


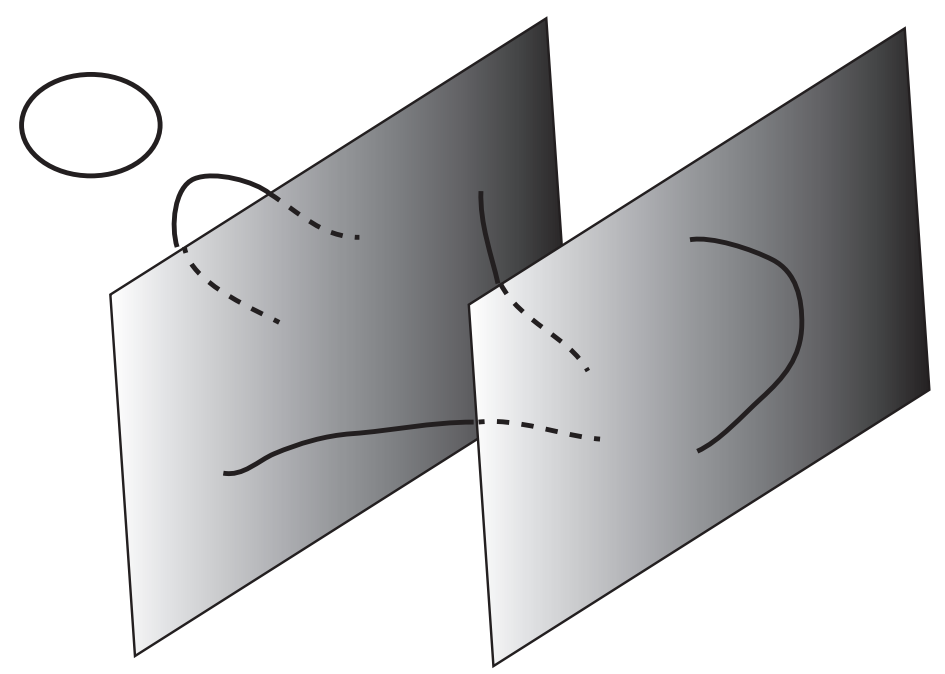

Fig. 1. D-branes: with open strings on the same surface and stretched between distinct surfaces. Open strings correspond to gauge particles, closed strings to gravitons.

not they contain closed strings. The machinery of dualities allowed these theories to be connected so that it was possible to view them as special Lorentz invariant limits of a single underlying theory ${ }^{7}$ — each of these consistent superstring theories is a possible quantum vacuum state of a deeper theory known, still tentatively, as $\mathcal{M}$-theory. A sixth possible Lorentz invariant vacuum was found, describing supergravity in 11 dimensional spacetime - this disparity in dimensions amongst the various solutions already suggests that at least some aspects of spacetime are not fundamental in the full definition of string theory. ${ }^{8}$ If one wishes to deal with four dimensions, then the six (or seven) residual dimensions must be made compact and hidden at low energies. ${ }^{9}$ Interestingly, in the case of the AdS/CFT duality, the equivalence holds between a non-compactified ten dimensional superstring theory and a four dimensional gauge theory. In other words, the claim is far more radical than the usual idea that oscillating strings wrapping around hidden extra dimensions of a compact space can reproduce ordinary low energy physics. It tells us that two distinct

7 Curiously, despite their enormous significance and power in physics, dualities remain relatively uncharted territory in philosophy of physics. For an initial detailed analysis of the general notion of duality, and its philosophical consequences, see Rickles (2011).

8 Other dualities (such as T-duality and mirror symmetry) push this conclusion even further by showing how topologically distinct spacetimes are identified by string theory. Again, see Rickles (2011) for more details.

9 This process radically increases the number of quantum vacua (giving rise to the 'string landscape'), though not all will possess features adequate to describe our own four dimensional world and, therefore - one can, quite legitimately in my view, invoke the anthropic principle here. 
theories can be subsumed into string theory such that they describe the same physics.

There is, of course, much more that could be said about the physics and mathematics of string theory. However, the pieces above ought to be sufficient to make enough sense of the AdS/CFT duality conjecture for the purposes of this paper. We turn next to an intermediate, though more general duality.

\section{Holography and the Gauge/String Duality}

Holography is widely expected to be a central feature of any future theory of quantum gravity and is deeply connected to issues of spacetime physics (see, e.g. Bousso (2005)). It is, then, a rare candidate for a Principle of Quantum Gravity. It states that for some description of a system of particles interacting gravitationally, we are able to describe the physics via a theory involving only the boundary of the spacetime region containing the system. ${ }^{10}$ The holographic screen contains all physical information for what occurs behind the screen (in 'the bulk') - here very schematically pictured in fig.2.

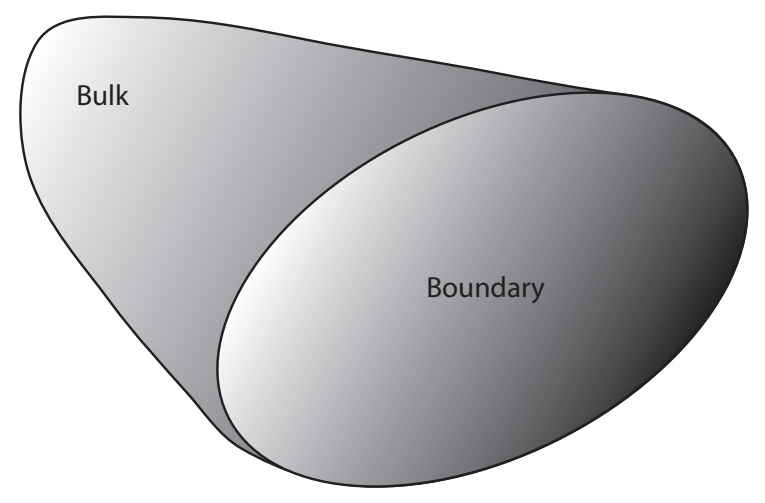

Fig. 2. The basic idea of the holographic principle: a theory defined on the boundary of the 'bulk' spacetime can describe the physics of systems within the bulk but off the boundary.

This is a rather general expression to be filled in by specific concrete instances. One gets close with the gauge/string duality, and finally gets a genuine concrete instance with the AdS/CFT duality.

\footnotetext{
$\overline{10}$ More generally it is the claim that the physics on the boundary is sufficient to describe the physics within the boundary.
} 
Duality refers, in general, to a correspondence between different regions of a parameter/moduli space. These can belong to what appear to be distinct theories. In particular, one can find regions in which the coupling constant is small in correspondence with regions in which the coupling is large. These values can belong to one and the same theory, in which case we might think of the self-duality as a simple gauge freedom. Or, in more interesting cases, such as the AdS/CFT duality, they can belong to seemingly very different theories involving very different structures. For example, S-duality relates Type 1 superstring theory to heterotic $S O(32)$ superstring theory. Recall that only a type 1 theory can contain open strings (and so gauge fields), but that the heterotic $S O(32)$ type contains only closed strings (and so gravity). S-duality establishes an equivalence between the strong coupling limit of one theory and the weak coupling limit of the other. In general:

$$
T\left(g_{s}\right) \equiv T^{\prime}\left(1 / g_{s}\right)
$$

Both types of theory are well-defined over all (non-negative) parameter values (i.e. the possible values of $g_{s}$ ), but their equivalence means that one can in principle write the complete physical story in terms of either. In our example this means that one can use a gauge theoretic description or a gravitational description. Hence the alternative name 'gauge/gravity' duality. It is as if 'choosing a theory' is tantamount to 'choosing a gauge'.

At weak coupling strengths (for which quantum effects are small) perturbation theory provides a tractable method of description and of computing physical quantities (such as correlation functions) within gauge theories and in string theory. At strong coupling (where one might expect to see new physics) this method is not usually practical and one has to resort to inexact techniques. The gauge/string duality allows one to employ an exact duality (between certain kinds of string and gauge theory) to circumvent the problems associated with strongly coupled gauge theories. One can set up a dual description in terms of a weakly coupled string theory, gaining access to exact information about the non-perturbative sector of the gauge theory. Alternatively, one can probe aspects of quantum gravity (in a restricted class of negatively curved backgrounds) using a weakly coupled gauge theory.

Of course, string theory began life as a theory of hadrons - with strings standing for flux tubes of field lines between quarks and anti-quarks - so we might quite naturally expect some kind of gauge-gravity link. Moreover, we know that string theory's major claim to fame is that it can account for both gravitation and the other interactions in the same framework. But string theory had too many problems to function well in this respect and was fairly quickly superseded by QCD, describing hadrons as having hard, point-like constituents, rather than the softer strings. QCD is an $S U(3)$ gauge theory, where the 3 
refers to the number of colours. QCD also possesses asymptotic freedom so that while the UV behaviour is computable (since the coupling constant decreases as the energy increases), the low energy behaviour requires far more approximate methods (such as lattice computations), since it is strongly coupled. However, string theory was able to describe certain aspects of hadrons quite well, and this empirical overlapping can be understood via the gauge/string duality.

The device connecting QCD and string theory is the large $N$ expansion (i.e. taking the number of colours or charges to be large) of 't Hooft which simplifies the theory by increasing the number of symmetries. The diagrammatic expansion of this field theory, in $\frac{1}{N}$, matches a free (open) string theory, with $\frac{1}{N}$ as the coupling constant. Hence, there is a relationship between large $N$ gauge theories and string theories. One can see in this way a typical duality symmetry situation: at weak string coupling, string theory can be used to discuss the strong coupling behaviour of gauge theory, and vice versa.

The Maldacena conjecture (also known as the AdS/CFT duality) is a concrete expression of this duality - though restricted to a supersymmetric cousin of QCD $^{11}$ ) - between large $\mathrm{N}$ gauge theory and string theory. It utilises the gauge/string connection, showing that an exact physical correspondence can be set up between string theoretic quantum gravity (in certain backgrounds) and large $N$ field theory in ordinary Minkowski spacetime. ${ }^{12}$ However, the spacetime dimensions of the two theories must be radically different (ten and four respectively) for the duality to establish itself. Naturally, one pressing task facing would-be interpreters of this duality is explaining this mismatch in dimensionality. In actual fact it has a fairly simple explanation: the spacetime dimensions on one side of the duality become internal degrees of freedom of particles on the other.

\section{What is AdS/CFT?}

Let us begin by getting clear on what the AdS/CFT (Anti-de Sitter/Conformal Field Theory) duality involves - an excellent, compact introduction is Klebanov (1998). The duality was first presented in Maldacena (1998). It amounts to the conjecture that a string theory in an anti-de Sitter space $A d S_{5}{ }^{13}$ pos-

\footnotetext{
${ }^{11}$ The conformal symmetry means, of course, that this supersymmetric theory is non-confining, since it is scale invariant: once one sets the coupling strength it remains at that strength independently of energy scale, unlike the QCD case. The supersymmetry stabilises the theory at high coupling.

12 Or, more precisely, the 4D conformal boundary of the string background.

${ }^{13}$ In fact, this must be a product space. Superstring theories are only well-defined in ten dimensions, so the remaining dimensions must be taken up by some other
} 
sesses equivalent physically observable properties to a conformal field theory defined on the (conformal) boundary $\partial \mathcal{S}_{\mathrm{AdS}_{5}}$ :

$$
\text { Strings on } \mathrm{AdS}_{5} \times \mathrm{S}^{5} \longleftrightarrow 4 \mathrm{D} \text { CFT on } \mathrm{AdS}_{5} \text { boundary }
$$

$A d S_{5} \times S^{5}$ is characterised by the metric:

$$
d s^{2}=\frac{r^{2}}{R^{2}} \eta_{\mu \nu} d x^{\mu} d x^{\nu}+\frac{R^{2}}{r^{2}} d r^{2}+R^{2} d \Omega_{5}^{2}
$$

The symbols here have the following interpretation:

$x^{\mu} \longmapsto 4$ coordinates of gauge theory

$\mathbf{r} \longmapsto$ radial dimension

$\Omega_{5} \longmapsto$ metric of unit 5 sphere $S^{5}$

$\mathbf{R} \longmapsto$ scale of spacetime (relative to strings) $=\frac{1}{\lambda_{t^{t} H o o f t}^{4}} l_{\text {string }}$

$\lambda_{\text {tHooft }} \equiv g_{\text {Yang-Mills }}^{2} N_{\text {colours }}$

$l_{\text {string }} \equiv \alpha^{\prime 1 / 2}$ ( $\alpha^{\prime}$ is the inverse string tension $)$

This metric corresponds to the horizon of a D3-brane. The intuitive picture (as laid out in Maldacena's original statement) can be discerned by imagining a stack of $N_{c}$ parallel D-branes (where the $c$ refers to the number of colours of the gauge theory). D-branes gravitate, with gravitational coupling strength determined by $g_{s}$. The warping in the geometry of spacetime is determined by $N_{c} g_{s}$. Given fixed $N_{c}$ we can tune the parameter $g_{s}$ across a range of values from very small to very large, corresponding to the amount of gravitational warping of spacetime geometry. This warping will be minimal, and the spacetime near flat, when $N_{c} g_{s} \ll 1$. In this case, there can be both open and closed strings,

space, $\mathcal{S}_{5}$ - this is an Einstein manifold (i.e. a solution of the Einstein field equations) of positive cosmological constant and it needs to be $S^{5}$ to get the symmetries of the gauge theory out right. Anti-de Sitter space is essentially like hyperbolic space with an additional time coordinate. It has a boundary and so one has to say what the boundary conditions are in any theory defined on this space. Naturally, this makes the theory very different to the usual philosophers' playthings since the existence of the conformal boundary implies that initial data and equations of motion are not sufficient to determine future evolution - one can have Earmanesque (non-superluminal) 'space invaders' coming in from spatial infinity. See Moschella (2005) for a very readable account of de Sitter and anti-de Sitter spaces. Of course, there is an immediate problem with this whole scheme that we have ignored: it is not realistic. Anti-de Sitter space has a negative cosmological constant, and in our universe it is apparently positive. There has been work on more realistic theories involving a dS/CFT correspondence (e.g. Strominger (2001)), but this is still very much work in progress. 
but with low coupling strength they will be virtually decoupled from each other. The closed strings that decouple from the open strings gives a picture of linearised, perturbative gravity. The open strings stuck to the D-brane, as we have seen (in the case of their low energy modes), are described by a gauge theory restricted to the D-brane (or D-brane stack).

If we increase the coupling strength so that $N_{c} g_{s} \gg 1$ then the gravitational effect of the D-branes on the spacetime metric becomes non-negligible, leading to a curved geometry and, in fact, a black hole geometry (or a black brane). By analogy with a standard Reissner-Nordstrom black hole, this geometry is $A d S_{5} \times S^{5}$ (cf. Horowitz and Polchinski (2009, p. 174)). Of course, strings sitting near the event horizon will be red shifted from the point of view of distant observers, and so will appear to have low energies. In the limit of low energies (ignoring massive states) the near-horizon strings decouple from the strings on the (flat) conformal boundary. Putting this together, it follows that at weak coupling the physics is described by a gauge theory and at large coupling described by a string theory. Juan Maldacena (Maldacena, 1998) conjectured that there was a duality linking these two descriptions together, so that it was really one theory being viewed from different regions of parameter space. Let us unpack this some more.

In this account we see that there are two ways of representing D-branes, in terms of open and closed strings respectively:

(1) On the one hand we can view them as features of the open strings stuck to them (qua surfaces on which open strings end-i.e. D-branes proper).

(2) On the other hand we can think of the branes in less fundamental terms, as a kind of topological defect in spacetime geometry (essentially a black hole, or black brane) described by closed strings.

When $r \rightarrow 0$ (in the 'near-horizon limit') the first representation of branes is modelled by the $4 \mathrm{D}$ gauge theory, while according to the second representation the behaviour is modelled by a IIB supergravity theory (the low energy limit of type IIB string theory) on $A d S_{5} \times S^{5}$. However, in the $r \rightarrow \infty$ limit (that is, on the boundary) both representations (D-branes and black branes) are modelled by a free IIB supergravity theory.

The holographic nature of this duality is evident from the fact that one is dealing with boundary data in the string theory. It is the boundary data that delivers the gauge field theory. The gauge theory lives on the $r \rightarrow \infty$ conformal boundary of $A d S_{5}$, with the string theory defined throughout the $r<\infty$ interior, i.e the bulk. This radial dimension (a 5th spatial dimension in the case of the string theory) is converted into an energy scale in the field theory on the boundary such that events at distances far from the boundary correspond to IR processes and those near the boundary correspond to UV 
processes. Hence, this resolves the puzzle about the difference in dimensions of the two theories. The puzzle dissolves once one realises that these are not all spacetime dimensions in the two theories. The remaining five dimensions of the string theory that appear to be missing in the gauge theory are retained as 'internal' degrees of freedom of the gauge particles. ${ }^{14}$ Note that this also resolves a problem with the attempt to reconstruct the interior data from the boundary data (or vice versa) since local events on the boundary (i.e. observables that are close) can be far apart in the interior, but the energydistance anti-correlation can (at least partially) account for this behaviour.

The equivalence implies, of course, that the Hilbert spaces of these two quantum theories will be equivalent, which is very curious since they are built up from very different types of object: strings, branes, gravitons, etc. on the string theory side and local gauge-invariant functionals of the gauge fields and their derivatives on the other. The correlators of the two quantum theories must also be the same though the correlators of the Yang-Mills theory on the boundary of $A d S_{5}$ will be translated (in the dual theory) as gravitational (string) amplitudes restricted to specific boundary conditions. The observables of the gauge theory (local operators) will be defined at points and so will involve probing in the ultraviolet regime. The transformation of the radial direction into an energy or renormalization scale implies just that the operators live on the boundary (or at infinity in the gravitational/string theory language) - $c f$. Horowitz and Polchinski (2009, p. 173).

One often speaks of a 'dictionary' for translating one set of concepts into the other, and it is via this dictionary that the first empirical work using string theory has been carried out. This work puts 5D black holes to work in representing strongly coupled aspects of QCD, such as quark-gluon plasmassee Myers and Vázquez (2008) for a good review of this work.

What is immediately conceptually interesting about this is that all closed string theories contain gravity, but the boundary conformal field theory does not contain gravity. Indeed, it is defined on a flat 4D Minkowskian spacetime $\mathbb{M}^{4}$ which forms the boundary of $A d S_{5}$, the region at spacelike infinity, at which the curvature of the $A d S_{5}$ goes to zero and the radial coordinate goes

14 The full ten dimensional spacetime coordinates of the string theory appear in the Yang-Mills theory as ten bosonic fields split between six scalar field to describe D3brane motion (one of these being the radial direction, and the other five being angles in transverse spatial directions that come from the matrix description of the branes) and four vector fields describing the low energy modes of the open strings stuck to the flat spacetime volume traced out by the D3 brane. The five angles map onto the 5 -sphere component of the full product space while the Minkowski spacetime coordinates of the D3 brane (i.e. the worldvolume) and the radial direction map on to $A d S_{5}$. The symmetries are preserved between the theories by a mapping from the conformal symmetry of the Yang-Mills theory to isometries of the metric. 
to infinity. If correct, this is a stunning result: string theory (with quantum gravity) is describable by a fairly ordinary (conformal) field theory. Moreover, the duality links microscopic physics (particles) with macroscopic physics (cosmology). However, in what sense can spacetime be said to emerge from this dictionary between the theories?

\section{$5 \quad$ Emergence in what sense?}

If we are to speak of the emergence of spacetime in the philosophers' sense, then it must be the case that spatiotemporal physics is supervening on nonspatiotemporal physics. In other words, we ought to find that spacetime geometry (and perhaps topology and other structures) are not part of some 'deeper' theory, but arise as novel consequences, say when there are many degrees of freedom present (in which case the spacetime is a collective phenomenon) or very strong interactions operating in the deeper theory. ${ }^{15} \mathrm{~A}$ weaker option, still along the same lines, is that one kind of spacetime geometry supervenes on another different kind of spacetime geometry or structure.

The standard claim within AdS/CFT circles (see, e.g., Seiberg (2006)) is that the emergent structure is a space with gravity (which itself is linked to the string theory, which itself implies that strings are also emergent: see Horowitz and Polchinski (2009, p. 178)). The underlying theory from which space, gravity (and strings) emerge is a local 4D quantum field theory. So clearly, the sense in which space is emerging here corresponds to my weaker option: there is a theory with its own space (here flat 4D Minkowski space, though with conformal symmetry) and a higher dimensional theory containing gravity (and therefore possessing general covariance) in an AdS background is emerging from this in such a way that the local QFT provides a holographic projection of the higher dimensional theory: quantum gravity is derivable from gauge theory. (Notably, the time coordinate is preserved in the AdS/CFT duality, and so does not emerge like the spatial geometry.)

In the weak coupling limit of the QFT (the 4D Yang-Mills theory) the physics is in the UV sector, near the boundary. One cannot 'see' the higher dimensions from here. To do this one tweaks the coupling, which, as we have seen, corresponds to moving along the radial direction and altering the scale (towards the IR region). It is only at strong coupling that one 'sees' the higher

\footnotetext{
${ }^{15}$ Horowitz and Polchinski (2009, p. 178) speak of emergent symmetries in the sense of the symmetry being "completely invisible" in the underlying theory. Nathan Seiberg defines emergent spacetime as "not present in the fundamental formulation of the theory, but appear as approximate macroscopic concepts" (Seiberg, 2006, p. 163).
} 
dimension in the local QFT, and in this sense space can be said to emerge from the increased interactions and fluctuations caused by upping the coupling strength ( $c f$. Koch and Murugan (forthcoming, p. 17)). If one can treat the $4 \mathrm{D}$ space as an auxiliary artifice for constructing the 'real' theory, then one might be said to have a stronger notion of emergence here. However, an obvious problem that faces one is that the duality relation is formally symmetric, so it would apparently make just as much sense to say that the gauge theory emerges from gravitational theory as the other way around.

There is another sense of emergence of spacetime, this time connected to the general covariance (or diffeomorphism invariance) of GR. The gravitational theory is dual to a non-gravitational theory and their gauge symmetries differ: Dif $f_{A d S_{5} \times S^{5}}$ on the one hand (a spacetime symmetry that moves points of the manifold around) and $S U(N)$ on the other. This suggests that neither is a fundamental symmetry. However, the way the AdS/CFT duality is currently established treats the gauge theory as fundamental: at large $N$, as one increases the 't Hooft coupling strength (the renormalization group parameter) there is a shift to an effective theory describing quantum gravity (i.e. the string theory). Again, given the link between gravity (and its symmetries) and geometry, we can speak of this as emergent spacetime - though, again, in the weak sense of a spacetime structure (and symmetries) emerging from another spacetime and symmetries.

A potential problem here concerns the usage of an unphysical, auxiliary metric (determined by the boundary conditions at infinity). The quantum gravity theory (the string theory, that is) must have its asymptotic behaviour fixed by the gauge theory. We should be a little concerned that a background spatial metric enters in the boundary conditions at infinity since spacetime and strings are mathematically derived using this auxiliary metric. (cf. Horowitz (2005), p. 211). The gauge theory directly employs this unphysical, flat, metric which is fixed at the boundary but not elsewhere, where it can undergo quantum mechanical fluctuations between different geometries. Hence, there is a background metrical structure appearing, but also extreme background independence elsewhere. Moreover, it is only restricted to this boundary that the gravitational, string theory has a local realisation within the gauge theory (thanks to the energy-distance relationship). This implies that there are no local observables in the bulk spacetime, only on the boundary. Switching to the gravitational theory itself, we naturally find that these same local observables form part of its boundary data.

So this leaves us with the following picture: string theory, gravity, and spacetime are captured holographically. They are represented by correlation functions on a conformal boundary at infinity where the local observables live. Of course, we do not live at infinity but nonetheless our measurements seem to be localized. How does this come about in such a picture? We know, however, 
that GR has no local observables: locality has to be achieved relationally (or at least non-locally) by constructing a system of (physical) rods and clocks. Already this points to the non-fundamental nature of spacetime qua manifold, so we should not be surprised by these problems of locality. Indeed, in many ways, some of these deep ontological consequences of the duality have been known for some time. For example, as Misner wrote in 1957:

Before studying general relativity we have no notion of a metric, no theory of distance and time. We are therefore unable to imagine any idealized physical theory which would provide a theory of events to use in discussing general relativity. The theory of events must spring up within general relativity, not logically precede it. When a scalar such as $R(x)$ is considered as an observable in classical general relativity, the point $x$ represents an event and is in reality a complicated functional of other events which the observer used to define his location, and of the metric throughout a region containing both those reference events and the event associated with $x$. If we were prepared to discuss such an observable, we would use a notation that gave fair warning of the complexity of the computation it envisages, and of the large number of quantities on which it depends. When we write $R(x)$ we mean a comparatively simple mathematical object, the value of the curvature scalar at a point $\mathrm{x}$ for the metric under consideration. The point is not an observable in the classical theory. Consequently, $R(x)$ is not an observable functional of the metric, nor is $g_{\mu \nu}(x)$, nor is the value of any scalar or tensor function at $x$. If $\phi$ is any function of points defined by the metric then we expect no corresponding operator $\phi(x)$ to be constructible in the quantum theory of general relativity. (Misner, 1957, p. 499)

The notion of an event in generally relativistic physics is not modelled by a point. Rather we ought to think of it as a complex, functional of physical quantities that serve to define a location. Hence, even classical general relativity is itself a theory in which spacetime location is not an invariant notion. But one still needs to define local observables. This will also be a challenge, then, for a theory of quantum gravity. Many of these issues have been well discussed in the context of canonical quantum gravity for many decades, beginning with Bergmann's group and Misner. Indeed, it strikes me that here is an area in which string theorists and loop quantum gravity theorists ought to work together.

Thus, even if we are faced with the prospect of the non-fundamentality of spacetime, we have a conceptual framework ready and waiting to make sense of this notion. "Background independence" is often used to describe this notion, though this terminology still possesses some looseness. The point is, however, that at a fundamental level, our descriptions of physical phenomena do not involve spacetime coordinates. We knew this already of course. AdS/CFT duality does not really impact on this, though it does show us how 
one can use a background dependent theory to generate results about a dual background independent theory. This suggests that the distinction between background independence and dependence might itself be non-fundamental which would cast extreme doubt on the ubiquitous claims in the philosophical and physics literature that constructing quantum gravity hinges on producing a background independent theory.

Carlo Rovelli views the imposition of boundary conditions, fixing the geometry at infinity, as sweeping the real problems of quantum gravity under the carpet:

The way this fundamental issue [BI] is addressed in string theory is often indirect. For instance, attempts are made to describe the bulk quantum geometry of spacetime by using the ADS-CFT conjecture, thus trying to describe what we do not know (quantum gravity) in terms of conceptual tools that we control (flat-space quantum field theory on the boundary). Analogously, the string theory calculations of black hole entropy exploit the relation between the strong-coupling genuinely-gravitational regime of interest, and the weak-coupling regime where conventional flat-space tools can be used, and states can be counted. Again, string cosmology often addresses the highly non-Minkowskian geometry of early cosmology by an hypothesis, that sounds bizarre to relativists: an overall larger Minkowski space where everything happens.

In all these cases, instead of addressing the real problem, which is to learn how to do physics where background spacetime plays no role, the strategy is to try to circumvent the problem, bringing back the calculations to the familiar pre-general-relativistic conceptual framework. The reason of this, of course, is not lack of imagination or courage from string theorists. String theory gives glimpses and hints of how a genuine theory of quantum geometry could be, with general states having no Riemannian spacetime interpretation at all - like a general state of a quantum particle is not necessarily similar to a classical localized particle-but for the moment it is far from providing a complete coherent picture of quantum geometry. (Rovelli, 2011, p. 3)

However, until we have a better grasp on the notion of background independence and its role in physical theories Rovelli's objections are not decisive. It might be that the 'bulk background independence' of the AdS/CFT duality might be all that one requires. Moreover, the loop quantum gravity (defended by Rovelli) approach begins with a fixed auxiliary manifold in order to define its fundamental degrees of freedom, so why not allow the same crutch (the auxiliary metric at infinity) to the string theorist? 


\section{Redundancy, Representation, and Realism}

In the discussion following Nathan Seiberg's talk on emergent spacetime, Shenker speaks up in "defence of redundancy" (Seiberg (2006), p. 179). I think he is right to draw attention to the latitude in representation in this context. Shenker points out that building redundancy into a representation can be very useful since different aspects of the physics become more explicit or transparent in different gauges. He suggests that it is too much to ask of a theory to do everything "in one presentation" (ibid.). This might be a very good suggestion, though it does somewhat conflict with a realist mindset. The realist will seek something that unifies the multiple descriptions of the different gauges.

The notion of a 'dictionary' connecting two theoretical descriptions of the same physical behaviour is telling. An anti-realist would find no problem with any of this. It is no different that computing in position versus momentum space, for simplicity of calculation. If one views the languages of gravity and gauge theory as not properly referring, then one is not going to find much to be puzzled about. I will disregard this approach, and assume we want to interpret physics in the sense of providing the possible worlds of a theory. Oriti (ed., p. 229) distinguishes three interpretive options that one could adopt with respect to the emergence of spacetime from AdS/CFT, the third of which corresponds to an anti-realist stance:

- The 4D spacetime of the boundary gauge theory is real, with the bulk spacetime appearing as an auxiliary construction.

- The 10D spacetime of the bulk string theory is real.

- The AdS/CFT is a purely mathematical device with no implications for reality.

Oriti (rightly, I think) points out that the first option roughly corresponds to what most string theorists believe. Horowitz and Polchinski (2009), in response to Oriti, counsel us to understand dualities as a change of variables. But if this is the case, as I think it is, then what sense does their claim that spacetime is emergent have? However, they also note that the AdS/CFT correspondence is a little different from other dualities in that the gauge theory side is exactly understood, but the string theory side is only approximately understood (via the gauge theory in fact). Thus they write:

In the AdS/CFT case, the situation may not be so symmetric [as other dualities - DR], in that for now the gauge side has an exact description and the string/gravity side only an approximate one: we might take the point of view that strings and spacetime are "emergent" and that the ultimate precise description of the theory will be in variables closer to the CFT form. 
"Emergent", however, is not the opposite of "real": most phenomena in nature are emergent, but nevertheless real. In particular, since we experience gravity, it would be this emergent description that is real to us. [Horowitz and Polchinski (2009, p. 230)]

This is a fair point. It is perfectly consistent, especially given the fact that the parameter governing the emergence is the coupling strength, to view the string theoretical description emerging from the field theory description in much the same way as chemical properties emerges from the quantum mechanical description. The standard low energy physics (including general relativity and, so the hope goes, the standard model) is then delivered by the string theory (after compactification of the 10-4 dimensions).

The fact that the two theories possess the same symmetries and that there is a smooth transformation mapping one to the other (the duality mapping) would lead a structuralist about scientific theories quite naturally to the conclusion that this is one physics being multiply represented. The fact that the two theories appear to be structurally very different might appear to be a problem at first sight: it looks like an example of structural underdetermination. And indeed it is. However, there is deep structure (the symmetries) that the two theories have in common. This characterises a deeper structure, not et fully understood.

There are surely lessons for the debate over scientific realism here. In particular, I think structural realism is well equipped to provide a sensible answer the the ontological mess that we find in the physics literature. We have, with the AdS/CFT duality, a situation in which two seemingly very different theories describe the same physics. We are used to this from examples of underdetermination, of course. In fact, I would say that dualities of this kind give us a response to Earman's challenge (or what (Lyre, 2011, p. 236) labels the "problem of missing examples":

Are there interesting cases of empirically indistinguishable theories? ... Here I find the philosophical literature disappointing [but] what we have is a shortcoming of the philosophical literature and not a failure of the underdetermination thesis. (Earman, 1993, p. 31)

Earman was absolutely right, and dualities provide interesting cases missing from the philosophical literature. They are especially interesting because the theories differ at a fairly deep, structural level, differing in such things as the number of spacetime dimensions, their symmetries, and so on. We have identifications between (quantum) gauge theory and (quantum) general relativity. ${ }^{16}$

$\overline{16}$ This clearly goes way beyond the example of structural underdetermination (or, more generally, mathematical overdetermination) that Lyre (2011, p. 245) isolates, namely different approaches to classical gravity (in which the gauge group differs). 
This might seem to pose a fatal blow to the structural realist programme, as Lyre argues, offering an example of structural underdetermination. We have a case where the empirical content is (effectively) identical, and yet the extraempirical content differs at a structural (not just the object-based) level.

The AdS/CFT example is of just this sort, but I do not see that it need be fatal to structural realists. One could adopt the same move as in underdetermined individualistic, object-base ontologies: identify the invariant core. Of course, this way triviality threatens. But I say that it in fact provides a methodology for scientific discovery: identify common structures between theories or structures and then try to understand this common structure as another deeper, broader theory. This is just what we find in the case of the duality between electricity and magnetism which leads us to the deeper electromagnetic field. Of course, identifying the invariant, unifying core in the case of string theory requires the next step in that theory to be taken. In a real sense, we still don't know what string theory is, though the AdS/CFT provides a window giving a limited view.

\section{Conclusion}

I have briefly introduced the AdS/CFT correspondence and the argument that is often presented to reveal the emergence of spacetime geometry. I argued that though it points to a kind of background independence within string theory, conceptually it is not really so novel and amounts to the basic lesson of general relativity: spacetime geometry and locality are not a fundamental features of reality. The duality is of a kind found in GR in another respect: neither picture related by the duality grasps the 'real' physics in a fully invariant fashion and one can transform between descriptions. In this case the coupling strength smoothly takes one between the descriptions. As the renormalization scale is increased one gets a new effective description. The question is: which parts of both descriptions are physical and which unphysical? That is ultimately a question for a deeper theory to decide. There are clear links to interesting philosophical issues about fundamentality, levels, and the like, but we save

However, it does point to the correctness of Lyre's claim that "we must be able to identify the structural core of the theory of gravity with some structure beyond the gauge symmetry group structure, such that the gauge group structure itself turns out as surplus" (p. 246). Note that Lyre doesn't agree that the paucity of examples Earman mentions is a shortcoming merely of the philosophical literature, writing: "To those, who do not share my doubts, I recommend to face Earman's challenge and come up with a more impressing list of cases" (p. 246). I contend that duality symmetries - of which many can be found with broadly similar properties vis-à-vis underdetermination - offer ideal candidates. 
these for another paper.

\section{References}

Bousso, R. (2002). The holographic principle. Reviews of Modern Physics 74: $825-874$.

Earman, J. (1993). Underdetermination, realism, and reason. Midwest Studies in Philosophy 18: 19-38.

Elvang, H. and J. Polchinski (2003). The quantum Hall effect on $\mathbb{R}^{4}$. Comptes Rendus Physique 4(3): 405-417.

Koch, R. and J. Murugan (forthcoming). Emergent spacetime. In J. Murugan, A. Weltman, and G. F. R. Ellis (eds.), Foundations of Space and Time: Reflections on Quantum Gravity. Cambridge University Press, 2012. http: //arxiv.org/abs/0911.4817

Horowitz, G. (2005). Spacetime in string theory. New Journal of Physics 7: 201-213.

Horowitz, G. and J. Polchinski (2009). Gauge/gravity duality. In D. Oriti (ed.), Approaches to Quantum Gravity: Toward a New Understanding of Space, Time, and Matter (169-186). Cambridge University Press, 2009.

Klebanov, I. R. (1998). TASI Lectures: Introduction to the AdS/CFT correspondence. In J. Harvey, S. Kachru, and E. Silverstein (eds.), Strings, Branes and Gravity (pp. 615-654). World Scientific, 2002.

Lyre, H. (2011). Is structural underdetermination possible? Synthese 180: 235-247.

Maldacena, J. M. (1998). The Large N limit of superconformal field theories and supergravity. Advances in Theoretical and Mathematical Physics 2: 231252.

Misner, C. W. (1957) Feynman quantization of general relativity. Reviews of Modern Physics 29(3): 497-509.

Moschella, U. (1998). The de Sitter and anti-de Sitter sightseeing tour. Séminaire Poincaré 1: 1-12.

Myers, R. C. and S. E. Vázquez (2008). Quark soup al dente: Applied superstring theory. Classical and Quantum Gravity 25(11): 1-12.

Norton, J. (2009). The inductive significance of observationally indistinguishable spacetimes. Unpublished manuscript: http://www.pitt.edu/ jdnorton/papers/Obs_Equiv.pdf.

D. Oriti, ed. (2009). Approaches to Quantum Gravity: Toward a New Understanding of Space, Time, and Matter. Cambridge University Press.

Polyakov, A. M. (1999). The Wall of the cave International Joural of Modern Physics A 14(5): 645-657.

Randall, L. and Sundrum, R. (1999). Large mass hierarchy from a small extra dimension. Physical Review Letters 83(17): 3370-3373.

Rickles, D. (2011). A philosopher looks at string dualities. Studies In History 
and Philosophy of Modern Physics 42(1): 54-67.

Rovelli, C. (2011). A critical look at strings. arXiv:1108.0868v1.

Seiberg, N. (2006). Emergent spacetime. In D. Gross, M. Henneaux, and A. Sevrin (eds.), The quantum structure of space and time (pp. 163-178). World Scientific, 2007.

Strominger, A. (2011). The dS/CFT correspondence. Journal of High Energy Physics 10: 034.

Witten, E. (1998). Anti-de Sitter space and holography. Advances in Theoretical and Mathematical Physics 2: 253291. 